\title{
Precursory signals of Forbush decreases with and without shock wave
}

\author{
Dimitra Lingri ${ }^{\circledR 1}$, Helen Mavromichalaki', Maria Abunina ${ }^{(2}$, Anatoly V. Belov ${ }^{\circledR 2}$, \\ Eugenia A. Eroshenko ${ }^{(12}$ \\ Correspondence \\ ${ }^{1}$ Faculty of Physics, National and Kapodistrian University of Athens, Greece, \\ dlingri@phys.uoa.gr, emavromi@phys.uoa.gr \\ 2 Pushkov Institute of Terrestrial Magnetism, lonosphere and Radio Wave Propagation (IZMIRAN), \\ Moscow, Russia, \\ abunina@izmiran.ru, abelov@izmiran.ru,erosh@izmiran.ru
}

\section{OPEN ACCESS}

This work is published under the Creative Commons Attribution 4.0 International license (CC BY 4.0) Please note that individual, appropriately marked parts of the work may be excluded from the license mentioned or may be subject to other copyright conditions.

If such third party materiat is not Unser the creaty materiatis not under the Creative Commons license, any copying, editing or public reproduction is only permitted with the prior consent of the respective copy right owner or on the basis of relevan legal authorization regulations.

\section{Keywords}

cosmic ray intensity; Forbush decreases; neutron monitors; precursory signals

\begin{abstract}
Many previous studies have shown that before the beginning of a Forbush Decrease (FD) of the cosmic ray intensity, a precursor signal can be observed. All these surveys were focused on FDs that are associated with a sudden storm commencement (SSC). In this work we demonstrate that precursors could also be observed in events without a SSC that is determined by an abrupt increase of the interplanetary magnetic field. The type of precursory signals and their diversity among the events are the main purpose of this study. We try to figure out similarities and differences on the signals and the associated events from both categories in the last fifty years, from 1969 to 2019, using the same selection criteria of the under investigation FDs. Simultaneously the orientation of the upcoming solar disturbances in comparison to the way they configure the increase of the interplanetary magnetic field and create these signals are discussed.
\end{abstract}

\section{Introduction}

Sudden decreases that are often observed in cosmic ray (CR) intensity were first studied by Scott Forbush (1937) and from then they are called as Forbush decreases (FDs) (Forbush 1954). In most cases, a shock wave generated by a Coronal Mass Ejection (CME) expanding from the Sun to the interplanetary medium, compresses the Earth's magnetophere and a sudden storm commencement (SSC) is created (Cane 2000). So, the FD of the CR intensity starts simultaneously with the SSC record.

But there are many recorded FD events, the starts of which are not connected with a SSC. Nowadays they are assumed to be generated by high speed solar wind streams (HSSWSs) from coronal holes (CHs), although there are cases when they are related with more intense solar expanding phenomena, like solar flares, CMEs etc. In these phenomena the onset is defined by a sharp increase in the value of the interplanetary magnetic field (IMF). These events are more gradual, smoother and with a smaller amplitude than the FDs with SSC (Melkumyan et al. 2019).

The main purpose of this study is to find out if a precursory signal could also be observed, when the Earth is outside of the front of the upcoming shock. Around the start of a FD, pre-decreases and pre-increases of the CR intensity, combined with changes in the first harmonic of the anisotropy at the equatorial plane $\left(\mathrm{A}_{\mathrm{xy}}\right)$ may appear some hours before the start of the event and can be useful for 
forecasting the upcoming disturbance (e.g. Papailiou et al. 2012; Lingri et al. 2016b; Tortermpun, Ruffolo, and Bieber 2018; Lingri et al. 2019 and references there in). These CR intensity anomalies can be detected by applying data analysis of the neutron monitors (NMs) of the worldwide network. They can be observed from $20 \mathrm{~h}$ up to $1-2 \mathrm{~h}$ earlier than the start of the main phase of the Forbush decrease, which in most cases is important for the early detection of the event (e.g. Asipenka et al. 2009).

Especially, a pre-decrease is due to the "loss cone" effect, where NMs are connected with a low density region of cosmic rays come from downstream of the shock (Leerungnavarat, Ruffolo, and Bieber 2003). On the other hand, the CR particles reflected by the front of the stream are accelerated and recorded at the Earth as pre-increases (Dorman et al. 1995). The observed precursor sign for each event depends on the heliographic position of the solar source in combination with the Earth's position in the heliosphere (Kozai et al. 2016) and on the nature of the flow.

So in this work we try to answer the question if a SSC is necessary to be able to observe a precursor as it is considered until today and if it is not, which are the similarities and the differences between the two kinds of events. NMs data from the last fifty years have been examined (19692019) and FD events for cosmic rays of $10 \mathrm{GV}$ rigidity characterized by amplitude, corrected for the geomagnetic effects, greater than $2 \%$ were selected. The cosmic ray anisotropy $\mathrm{A}_{\mathrm{xy}}$ before the start of the FD was chosen to be greater than $0.8 \%$ and the interplanetary space conditions to be quite undisturbed. In Section 2 the data sources, the methodology that has been used and the selection procedure are presented. In Section 3 two characteristic events, one from each category with and without SSC that present precursory signals are analyzed Finally Section 4 contains discussion and new conclusions of this study.

\section{Data selection and methodology}

\subsection{Data collection}

In this work, the studied time period was chosen to cover four and half solar cycles (half of 20 , 21, 22, 23 and 24 solar cycle), from 1969 to 2019, as to have solar, cosmic and interplanetary data simultaneously with observations of the solar disk obtained from Geostationary Operational Environmental Satellites (GOES, https://www.ngdc.noaa.gov/stp/space-weather/solar-data/solar-features/solar-flares/x-rays/goes/xrs/, last accessed April 7, 2021). It is noticed that there are some data gaps around 1985-1992, where no continuous solar wind and/or interplanetary data exist.

Forbush Effects and Interplanetary Disturbances Database (FEID), (http://spaceweather.izmiran.ru/eng/dbs.html) have been used for the FDs and their characteristic solar and interplanetary parameters, calculated for the rigidity of $10 \mathrm{GV}$, which is close to the effective rigidity of most NMs, by using the Global Survey Method (GSM) (Belov et al. 2018). Finally, the OMNIWeb site (https:// omniweb.gsfc.nasa.gov, last accessed April 7, 2021) for the interplanetary and geomagnetic parameters was used.

Cosmic ray data were taken from the high resolution neutron monitor database (NMDB, https:// www.nmdb.eu/, last accessed April 7, 2021; Mavromichalaki et al. 2011) and from the worldwide cosmic ray center of Nagoya University (http://cidas.isee.nagoya-u.ac.jp/WDCCR/, last accessed April 7, 2021). Some criteria for the selected NMs have been used, as the rigidity of the NM station to be smaller or equal to $3 \mathrm{GV}$ and the location to be at an altitude lower than $1000 \mathrm{~m}$, as their measurements are affected by the cosmic ray anisotropy and density fluctuations. The sub-polar NM stations are not taken into account in this study, as they are affected by the north- south anisotropy $\mathrm{Az}$ (Belov et al. 2017a, 2017b). It is noticed also that the number of the used NM stations varies from time to time because new stations are founded and some others are shut down. However, in average for each studied event data were extracted from a number of around 20-25 NM stations. 


\subsection{Methodology and selection criteria}

For the selection of the events under investigation the Global Survey Method (GSM) was used, which unifies the simultaneous observations of cosmic ray intensity from terrestrial monitors and the anisotropy's variations for particles with magnetic rigidity of $10 \mathrm{GV}$, which is close to the effective rigidity of the most neutron monitors (Belov et al. 2018).

The criteria that were used, are similar to those of Lingri et al. (2019), but instead of the amplitude of FD at $10 \mathrm{GV}$ rigidity, we use the amplitude corrected for the magnetospheric effect by the Dst index (MagnM), as was calculated at the same rigidity (Belov et al. 2018; http://spaceweather. izmiran.ru/dbs/fds/full-list-parameters-eng.pdf, last accessed April 7, 2021). MagnM is more specific and helpful to avoid some magnetospheric alterations in the cosmic ray intensity capture. Which of them will be greater depends on the existing geomagnetic circumstances. So, the pre-conditions that are chosen for this detailed study of FDs with a precursory signal before are:

I) FDs to be (or not) associated with an SSC.

II) The MagnM to be equal or greater than $2 \%$. As precursors are about $2 \sigma$ of the FD's amplitude, this value helps us to avoid cosmic ray background fluctuations to be falsely characterized as precursor.

III) The $A_{x y}$ component of CR anisotropy one hour before the onset to be greater that $0.8 \%$. As the mean value of $A_{x y}$ is around $0.52 \%$, the value of $0.8 \%$ allows us to collect as many candi-

date events as we can, whose diurnal variations amplitude is close to the $A_{x y}$ calculated values (Belov et al. 2017a).

There is the preference for the interplanetary environment to be quiet or with small fluctuations before the start of the event, so the precursors to be clearly detected. If the interplanetary magnetic field is disturbed before the start of a FD, we exclude the event from our investigation. In FEID CR intensity variations are recorded, which means that there are also some Forbush increases and Ground Level Enhancements (GLEs) for example, which may have an amplitude greater than $2 \%$ but we do not want them in our database and they have to be excluded.

Finally it has to be noted that we avoid to examine FDs, which reached their minimum amplitude two to three days after their start, because in these cases we cannot characterize the fluctuations before the onset of the FD as precursors. We should notice that in the events where an SSC does not define the start of a FD, we have to put by hand the onset of each FD that in our study is determined by the first hour when an abrupt increase of the IMF starts to be recorded.

For the determination of the precursory signals we use the Ring of Station Method - RSM, which turns the geographical longitudes to asymptotic and determines the reception cone of each station and the diurnal anisotropy variation which is recorded in each neutron monitor in connection with the asymptotic longitudes (for details see Abunina et al. 2020). RSM was used to find out the possible existence of a precursor, by plotting the asymptotic longitudinal cosmic ray distribution diagrams, in which NM data are captured which were hourly corrected for pressure. The Tsyganenko96 model for the cut off rigidity is used to calculate the asymptotic directions of each station (Tsyganenko and Stern 1996).

\section{Selected Forbush decreases}

FEID database includes more than 6000 events that took place in the studied time period 19692019. From them, if we apply the criterion MagnM to be greater than 2\%, 1173 FDs remain to be examined. When all criteria are taken into account, 153 FDs associated with SSC and 74 FDs without a SSC connection remain to be investigated. About the percentage of $30 \%$ of the events of 
each category could be studied for precursors, as we examine each FD separately for simultaneous $\mathrm{CR}$, solar and interplanetary data and for the way of how the CR intensity changes throughout the time. In the next section one characteristic event with precursors for each category is analyzed.

\subsection{FD associated with SSC - The event of 15.02.2014}

The event occurred on 15 February 2014 at 13:16 UT is used as an example in the category of the FDs that are associated with a shock wave. The events of this category have been analyzed in detail also in previous studies (e.g. Lingri et al. 2016a; Lingri et al. 2019). It was associated with a halo CME with velocity of $533 \mathrm{~km} \mathrm{~s}-1$, that created a rapid increase in the solar wind speed and also in the IMF, which reached the value of $16.2 \mathrm{nT}$. The produced FD's amplitude reached the value of $4.0 \%$ for CRs of $10 \mathrm{GV}$ rigidity and significant variations of the Axy component took place (figure 1). The Axy and Az components of the anisotropy were increased after the SSC arrival. Moreover the Axy changed its direction significantly after the arrival of the main ejecta and of the associated magnetic cloud (orange arrows in figure 1). Finally, it was not associated with a geomagnetic storm, as the Dst index reached the value of $-37 \mathrm{nT}$.

From the figure 2 a precursor signal is observed before the shock wave arrival. A pre-increase precursor was observed from 10 to $2 \mathrm{hrs}$ before the FD at an asymptotic longitude range between $110^{\circ}$ and $320^{\circ}$. In the meantime a pre-decrease signal started to be recorded by the NMs about 4 hrs before the SSC in a range of $40^{\circ}-130^{\circ}$. This means that many stations show a significant increase of the recorded variations and a few ones present a minimum.

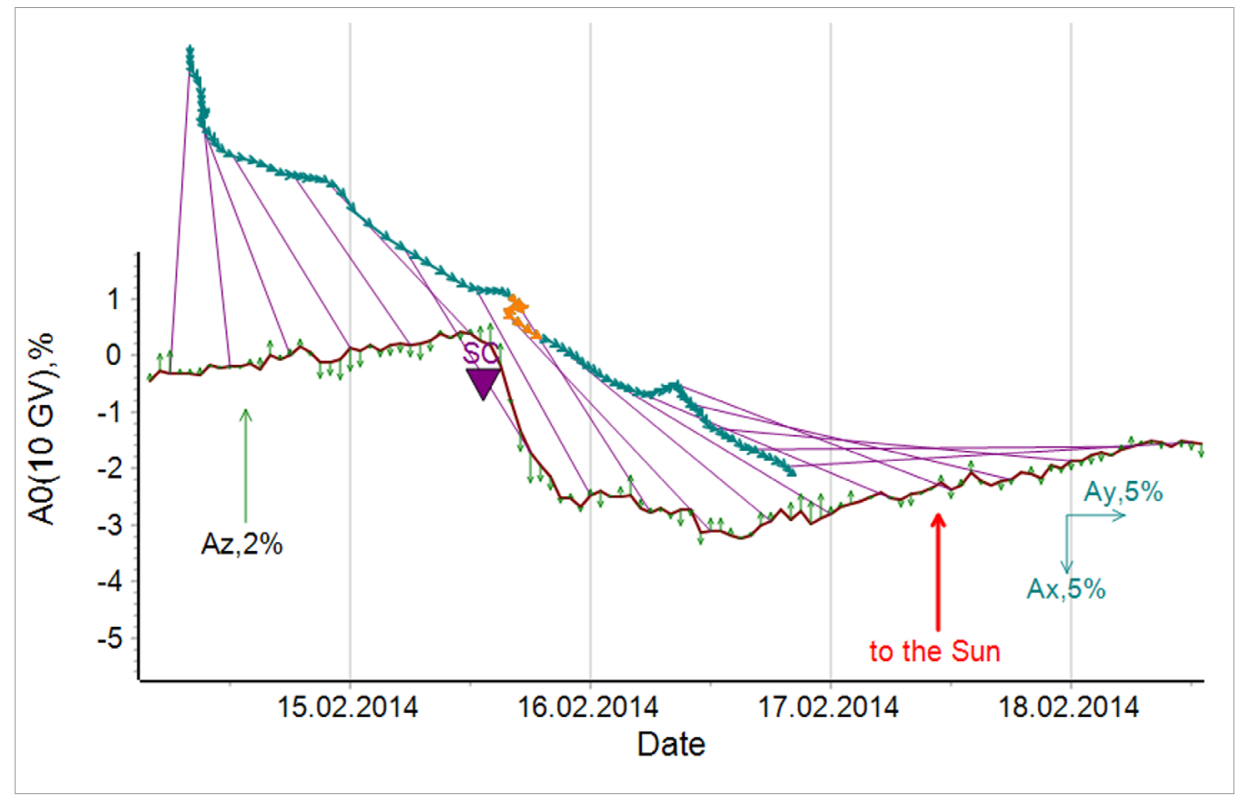

Figure 1: The anisotropy variation on the equatorial plane ( $A x y$ - blue vectors) and of the $z$ component ( $\mathrm{z} z$ - green vectors) during the time period of the event of 13-18 February 2014. The red line represents the CR intensity variations. The purple lines connect the same points in time on the two curves of the blue and the red lines. 


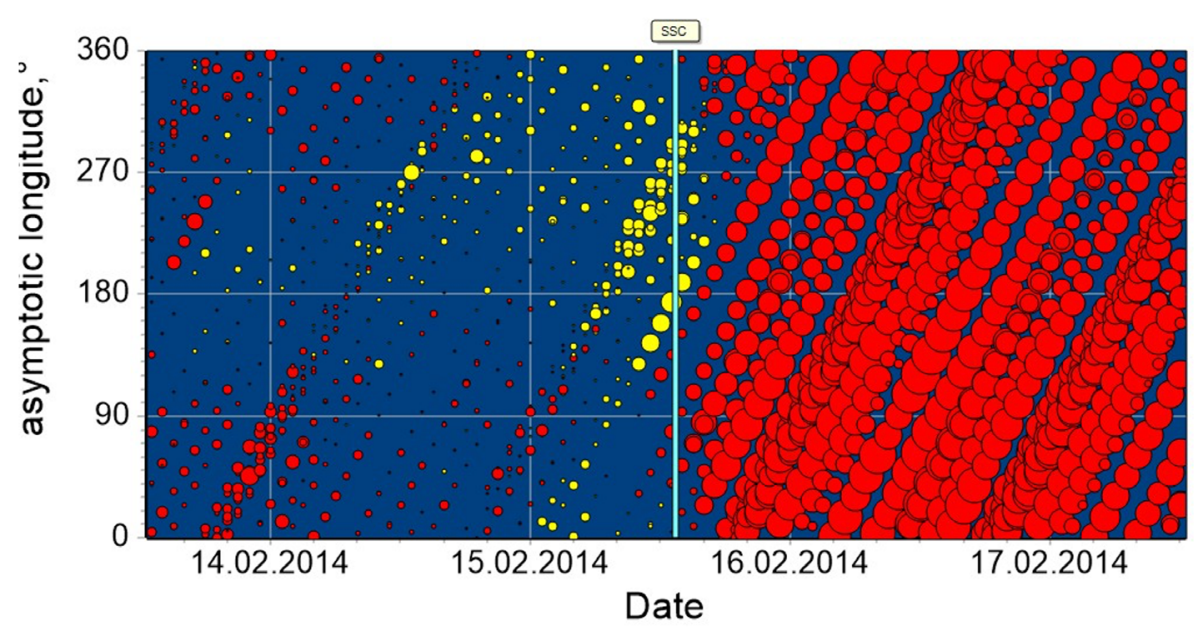

Figure 2: The asymptotic longitude of each station versus the time of the FD on 15 February 2014 is presented. The red circles represent decrease of the $C R$ intensity, while the yellow ones represent increase. The cyan vertical line indicates the shock arrival time. The size of its bubble is proportional to the hourly recorded cosmic ray amplitude of each station.

\subsection{FD without an association with SSC - the event of 21 October 2003}

Forbush decrease of 21 October 2003 at 14:00 UT is an interesting example of event not connected with a SSC, but with precursory signals. It is the first of a series of intense events that took place in October 2003 (figure 3), with the last one to be the greatest FD not only of the solar cycle 23, but also of the last fifty years. As it is shown in figure 3 this FD of 21 October 2003, in contrary to the next ones, started with an onset.

In 19 October the active region 10484 had just dawned on the observable from Earth solar disk and three large solar flares are erupted (2 M-flares and $1 \mathrm{X}$-flare). The solar wind velocity reached the value of $744 \mathrm{~km} \mathrm{~s}-1$ and IMF increased to $11.8 \mathrm{nT}$. It is generally considered that FDs without an association with a SSC have smaller amplitudes than the FDs that occur with a strong shock wave. This is not real and this specific event has an amplitude, corrected for geomagnetic effects, of $6.2 \%$ at the rigidity of 10 GV. Finally, a moderate geomagnetic storm was recorded, with the minimum of Dst index to be $-61 \mathrm{nT}$.

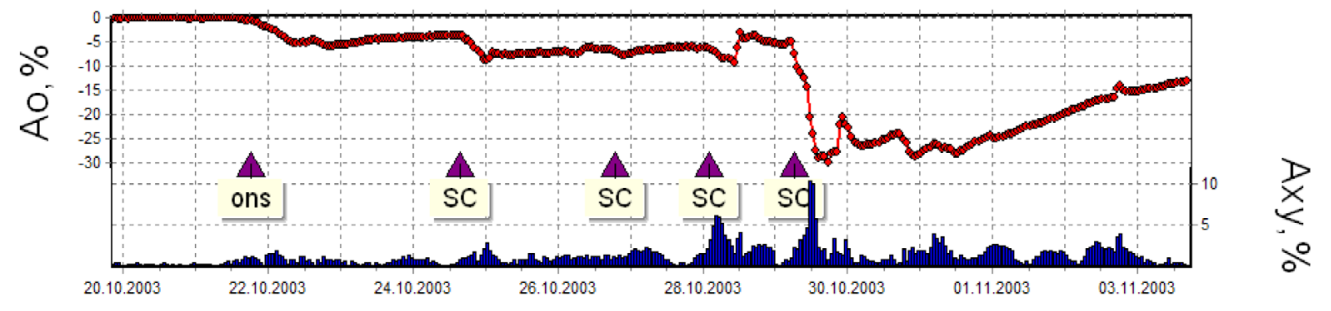

Figure 3: The $C R$ intensity $(A 0)$ (red line) and the anisotropy on the equatorial plane $A_{x y}$ (blue histogram) for the time period 20 October 2003 - 4 November 2003. 
An untypical pre-decrease precursor signal is observed before the onset of the FD. Pre-decrease sign started to be recorded by the NMs about $8 \mathrm{~h}$ before the onset of the FD and it ranged between $110^{\circ}-360^{\circ}$ (figure 4). It is noted that the untypical kind of precursors does not depend on the mechanism that generates the FD and is observed in both categories.

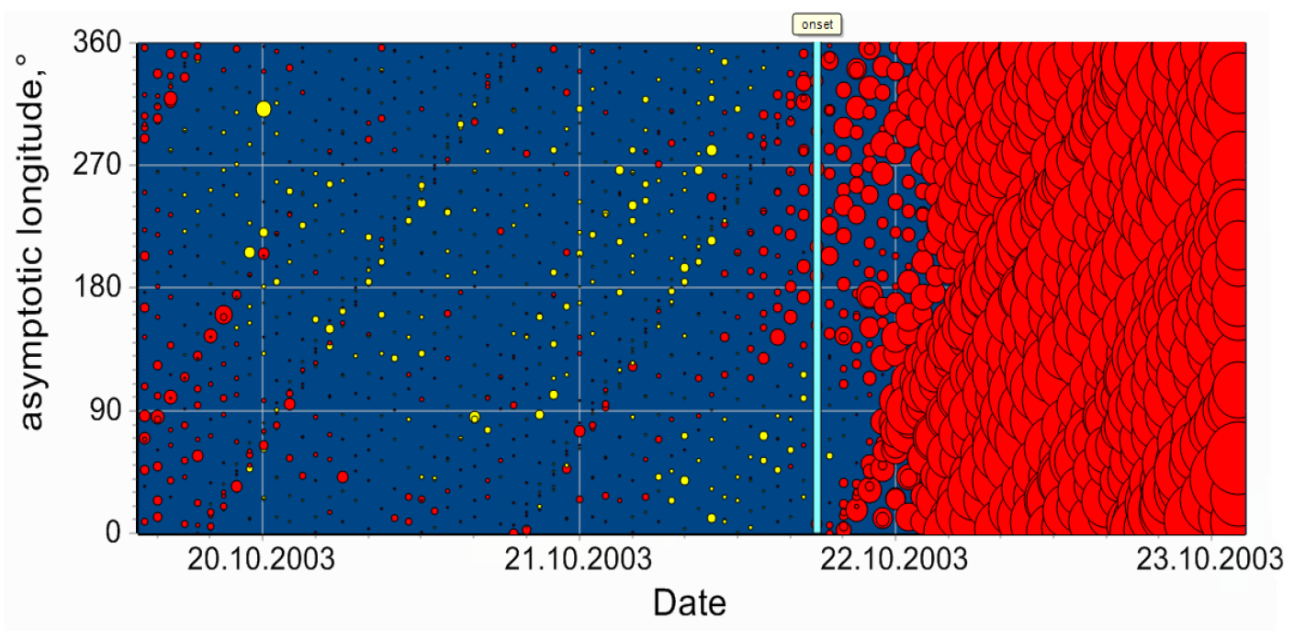

Figure 4: The asymptotic longitude of each station versus time (upper panel) of the FD on 21 October 2003. The red circles represent decrease in the CR intensity while yellow ones represent increase. Cyan vertical line is the onset time. The switching of yellow and red circles two days before the FD is the solar diurnal anisotropy record. The size of its bubble is proportional to the recorded amplitude of each station every hour.

\section{Discussion and conclusion}

From the study of the precursors of FDs that took place at the time period $1969-2019$ (solar cycles 20, $21,22,23,24)$ remarkable results were obtained. The most important and innovative of them is that indeed precursory signals could be observed before FDs which are not associated with the presence of a SSC in the upper magnetosphere. The two categories present similarities, mainly in the kind of precursor and the time period before the start of the FD where it appears. They present differences in the orientation of the solar phenomenon that generated the event and the shape of the precursory signal.

The events that are not associated with a SSC happened due to a phenomenon occurring in the eastern region of the solar disk and in some cases due to a coronal hole. On the contrary most FDs accompanied by a shock wave come from phenomena in the active regions occurring in the central and western zones of the solar disk.

Precursors are really anisotropic phenomena and can be observed as pre-decreases, pre-increases and in same events both of them are recorded (Lingri et al. 2019). It is the same in both categories, but in the case of the absence of a SSC, when a shock wave does not exist, pre-increases are rare as this phenomenon is directly connected with the front of the shock wave. The front swept out the cosmic ray particles and when there is a connection with the reception cone of the NM station a pre-increase is recorded.

It has to be noticed that typical and untypical precursors appear in both categories of FDs and they are not a point of the association or not with a SSC. In general for typical FDs without SSC, the asymptotic longitudes where the pre-decreases are observed are ranged between $\sim 0^{\circ}-190^{\circ}$ and starts about $18 \mathrm{~h}$ before the onset, while the few pre-increases appear in a range of $\sim 190^{\circ}-300^{\circ}, 8 \mathrm{~h}$ 
before the start of the event. On the other hand, the signals in FDs with a SSC are quiet narrower than those without a SSC, with the asymptotic longitudes where pre-decreases are observed ranging between $\sim 0^{\circ}-160^{\circ}$ and starting about $18 \mathrm{~h}$ before the SSC, while pre-increases appear in a range of $\sim 220^{\circ}-330^{\circ}$, $10 \mathrm{~h}$ before the start of the event.

The shape of the signal of a typical pre-decrease starts narrow and then expands as it goes close to the onset when there is no association with a SSC, while in the other category the range of the signal is about the same throughout the time. Finally, it has to be noticed that with the new criteria in both categories $\sim 70 \%$ of the studied events present precursory signals, while in previous studies, for the case of FDs associated with a SSC by using different criteria, the percentages were about 30\% and $\sim 47 \%$ (Papailiou et al. 2012; Lingri et al. 2019), respectively.

\section{Acknowledgments}

This research work was supported by the Hellenic Foundation for Research and Innovation (HFRI) and the General Secretariat for Research and Technology (GSRT), under the HFRI PhD Fellowship grant (GA. no. 14492). Special thanks to the colleagues of the NM stations (https://www.nmdb.eu/), last accessed April 7, 2021) for kindly providing the cosmic ray data used in this study in the frame of the High resolution Neutron Monitor database (NMDB) funded under the European Union's FP7 Program (contract no. 213007). Thanks are due to the IZMIRAN/FEID, ACE/Wind, OMNI and NOAA data centers.

\section{References}

Abunina, M., Belov, A.V., Eroshenko, E.A., et al., 2020, Solar Phys. 295, 69, DOI: https://dx.doi.org/10.1007/s11207-020-01639-7 Asipenka, A.S., Belov, A.V., Eroshenko, E.A., et al., 2009, Adv. Space Res. 43, 708, D0I: https://dx.doi.org/10.1016/j.asr.2008.09.022 Belov, A., Abunina, M., Abunin, .A., et al., 2017a, Geomagn. Aeron. 57, 389, DOI: https://dx.doi.org/10.1134/S0016793217040028 Belov, A., Abunina, M., Abunin, .A., et al., 2017b, Geomagn. Aeron. 57, 541, DOI: https://dx.doi.org/10.1134/S0016793217050036 Belov, A., Eroshenko, E., Yanke, V., et al., 2018, Solar Phys. 293, 68, D0I: https://dx.doi.org/10.1007/s11207-018-1277-6 Cane, H.V., 2000, Space Sci. Rev. 93, 55, DOI: https://dx.doi.org/10.1023/A:1026532125747 Dorman, L.I., Villoresi, G., Belov, A.V., et al., 1995, Nucl.Phys. B, Proc. Suppl. 39, 136, DOI: https://dx.doi.org/10.1016/0920-5632(95)00016-3 Forbush, S.E., 1954, J. Geophys. Res. 59, 525, DOI: https://dx.doi.org/10.1029/JZ059i004p00525

Kozai, M., Munakata, K., Kato, et al., 2016, Astrophys. J. 825, 100, DOI: https://dx.doi.org/10.3847/0004-637X/825/2/100 Leerungnavarat, K., Ruffolo, D., Bieber, J.W., 2003, Astrophys. J. 593, 587, DOI: https://dx.doi.org/10.1086/376408 Lingri, D., Mavromichalaki, H., Belov, A., et al., 2016a, Solar Phys. 291, 1025, D0I: https://dx.doi.org/10.1007/s11207-016-0863-8 Lingri, D., Mavromichalaki, H., Belov, A., et al., 2016b, in XXV ECRS 2016 Proceedings-eConf C16-09-04.3, https://arxiv.org/abs/1612.08900 Lingri, D., Mavromichalaki, H., Belov, A., et al., 2019, Solar Phys. 294, 70, DOI: https://dx.doi.org/10.1007/s11207-019-1461-3 Mavromichalaki, H., Papaioannou, A., Plainaki, et al., 2011, Adv. Space Res. 47, 2210. D0I: https://dx.doi.org/10.1016/j.asr.2010.02.019 Melkumyan, A.A., Belov, A.V., Abunina, M.A., et al., 2019, Adv. Space Res. 63, 1100, D0I: https://dx.doi.org/10.1016/j.asr.2018.10.009 Papailiou, M., Mavromichalaki, H., Belov, A., et al., 2012a, Solar Phys. 276, 337, DOI: https://dx.doi.org/10.1007/s11207-011-9888-1 Tortermpun, U., Ruffolo, D., Bieber, J.W., 2018, Astrophys. J. 852, L26, DOI: https://dx.doi.org/10.3847/2041-8213/aaa407 Tsyganenko, N., Stern, D., 1996, J. Geophys. Res. 101, 27187, DOI: https://dx.doi.org/10.1029/96JA02735

\section{Questions and answers}

Ludwig Klein: It looks like your FDs from the eastern solar hemispheres have no shocks visible at the Earth? Isn't the shock usually supposed to be broader than the driver?

Answer: Yes, it is true. The shock is broader than the driver, but as the shock moves inside the heliosphere, following the Parker spiral, the front of the shock does not reach the Earth's magnetosphere. So, the Earth intercepts the downstream region of the shock, which indeed is not observable at the Earth. 